\title{
Grade 3 well-differentiated neuroendocrine tumor of the rectum: a case report
}

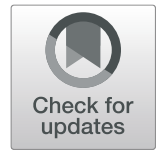

\author{
Misato Ito ${ }^{*}$ (D), Yasumitsu Hirano, Toshimasa Isii, Hiroka Kondo, Liming Wang, Masahiro Asari, Nao Obara and \\ Shigeki Yamaguchi
}

\begin{abstract}
Background: The 2019 revised World Health Organization (WHO) classification of tumors of endocrine organs classifies grade 3 gastroenteropancreatic neuroendocrine neoplasms (GEP-NEN G3) into well-differentiated tumors (NET G3) and poorly differentiated carcinomas (NEC G3). There are few reported cases of NET G3 occurring in the rectum.
\end{abstract}

Case presentation: A 71-year-old man complained of bright red blood in his stool. Total colonoscopy revealed a mass in the lower rectum. Pathologic examination yielded a diagnosis of group 1. Computed tomography revealed swollen paraintestinal lymph nodes and multiple liver metastases. We performed laparoscopic abdominoperineal resection not only to avoid the unbearable symptoms caused by tumor growth but to make a pathological diagnosis. The tumor measured $3.5 \times 2.8 \mathrm{~cm}$, and the pathological diagnosis was stage IV neuroendocrine carcinoma. He underwent chemotherapy with irinotecan plus cisplatin, followed by carboplatin plus etopside, but his disease did not respond to either regimen. Twenty-seven months after surgery, he died of his disease. Upon reexamination of the surgical specimen, the tumor was consistent with the 2019 WHO classification of NET G3.6

Conclusion: A definite diagnosis of NET G3 or NEC G3 must be made to determine the appropriate treatment strategy for patients with GEP-NEN G3. Further case reports and case series are needed to establish the optimal therapy.

Keywords: Neuroendocrine tumor G3, Colon, Rectum

\section{Introduction}

The 2019 revised World Health Organization (WHO) classification of tumors of endocrine organs classifies grade 3 gastroenteropancreatic neuroendocrine neoplasm (GEP-NENs) into well-differentiated neuroendocrine tumors (NET G3) and poorly differentiated neuroendocrine carcinomas (NEC G3). The concept of NET G3 was first described in the WHO 2017 classification of pancreatic tumors related to multiple endocrine neoplasia (MEN) syndromes. There are few reported cases of NET G3 occurring in the rectum, and there are currently no data on antitumor therapy for patients with metastatic colorectal NET G3.

\footnotetext{
* Correspondence: misatoito.saku@gmail.com

Department of Gastroenterological Surgery, Saitama Medical University International Medical Center, 1397-1, Yamane, Hidaka-City, Saitama-Pref, Japan
}

(c) The Author(s). 2020 Open Access This article is licensed under a Creative Commons Attribution 4.0 International License which permits use, sharing, adaptation, distribution and reproduction in any medium or format, as long as you give appropriate credit to the original author(s) and the source, provide a link to the Creative Commons licence, and indicate if changes were made. The images or other third party material in this article are included in the article's Creative Commons licence, unless indicated otherwise in a credit line to the material. If material is not included in the article's Creative Commons licence and your intended use is not permitted by statutory regulation or exceeds the permitted use, you will need to obtain permission directly from the copyright holder. To view a copy of this licence, visit http://creativecommons.org/licenses/by/4.0/.

\section{Case report}

A 71-year-old Japanese man had a known colonic polyp, but he did not have it rechecked for 7 years until he began to experience hematochezia. Colonoscopy revealed the tumor of the rectum, and he was referred to our hospital. He had no significant medical history. His father had suffered from lung cancer and a testicular tumor; however, there was no evidence of MEN in his family history. He did not smoke or drink alcohol, and he had no allergies.

A mass was palpable on rectal examination, forming a semicircle at the posterior wall of the rectum. Laboratory analysis revealed a total peripheral leukocyte count of $7100 / \mathrm{mm}^{3}$, with normal tumor markers (carcinoembryonic antigen [CEA], $2.3 \mathrm{ng} / \mathrm{mL}$; cancer antigen 19-9 [CA 19-9], 19.6 U/mL). His height was $165.5 \mathrm{~cm}$, and his weight was $57.9 \mathrm{~kg}$, giving a body 
mass index of $21.14 \mathrm{~kg} / \mathrm{m}^{2}$. Total colonoscopy revealed a mass in the lower rectum (Fig. 1). The tumor described a quarter circle around the rectum and was in contact with the anal canal. Pathologic examination of a biopsy specimen yielded a diagnosis of group 1 (ulcerative lesion with generated epithelium). Computed tomography (colonography) revealed a tumor in the lower rectum, suspicious for invasion of the right levator ani muscle, swollen paraintestinal lymph nodes, and multiple liver metastases (Fig. 2). We judged that the liver metastases were unresectable because they occurred in both lobes with adjacent to major hepatic vein. There were no metastases to the lungs or other structures. Magnetic resonance imaging confirmed the presence of swollen right lateral lymph nodes (Fig. 3).

We performed laparoscopic abdominoperineal resection of the rectum and lymph node dissection, with resection of the right pelvic plexus not only to avoid the unbearable symptoms caused by tumor growth but to make a pathological diagnosis of the rectal tumor. The operative time was $279 \mathrm{~min}$, and the intraoperative blood loss was $200 \mathrm{~mL}$. The tumor was located in the lower wall of the rectum and measured $3.5 \times 2.8 \mathrm{~cm}$ (Fig. 4).

Histological examination showed the rectal tumor with subserosal invasion (pT3) involving venous and lymphatic invasion. Immunohistochemical staining revealed that diffuse staining for synaptophysin and chromogranin A, and focal staining for CD56, and MIB-1 index was over $20 \%$. The results of CK7, CK20, and S-100 protein were negative. The patient's Ki67 index was 25 percent. The pathologic diagnosis of the day was neuroendocrine carcinoma, stage T3N3M1(H2), according to the $2010 \mathrm{WHO}$ classification of tumors of

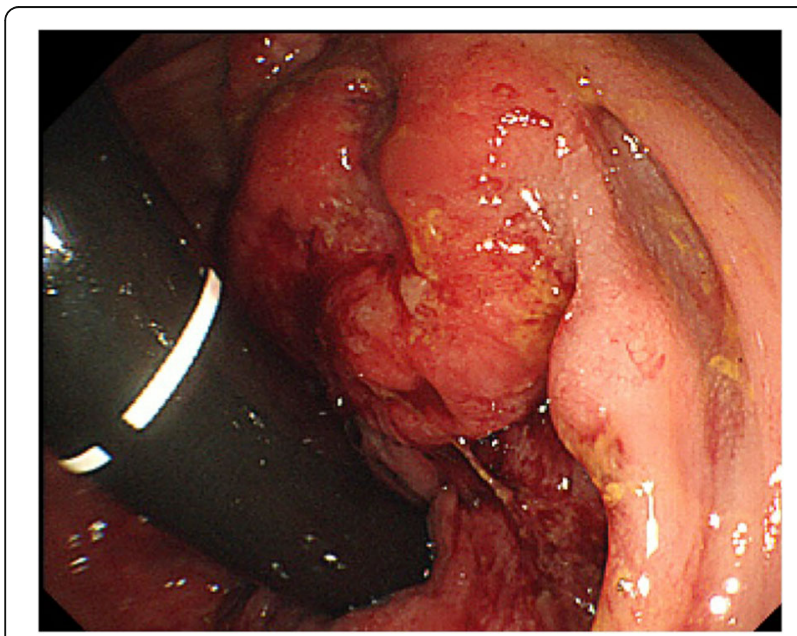

Fig. 1 Colonoscopy image showing a mass in the lower rectum, describing a quarter circle and in contact with the anal canal
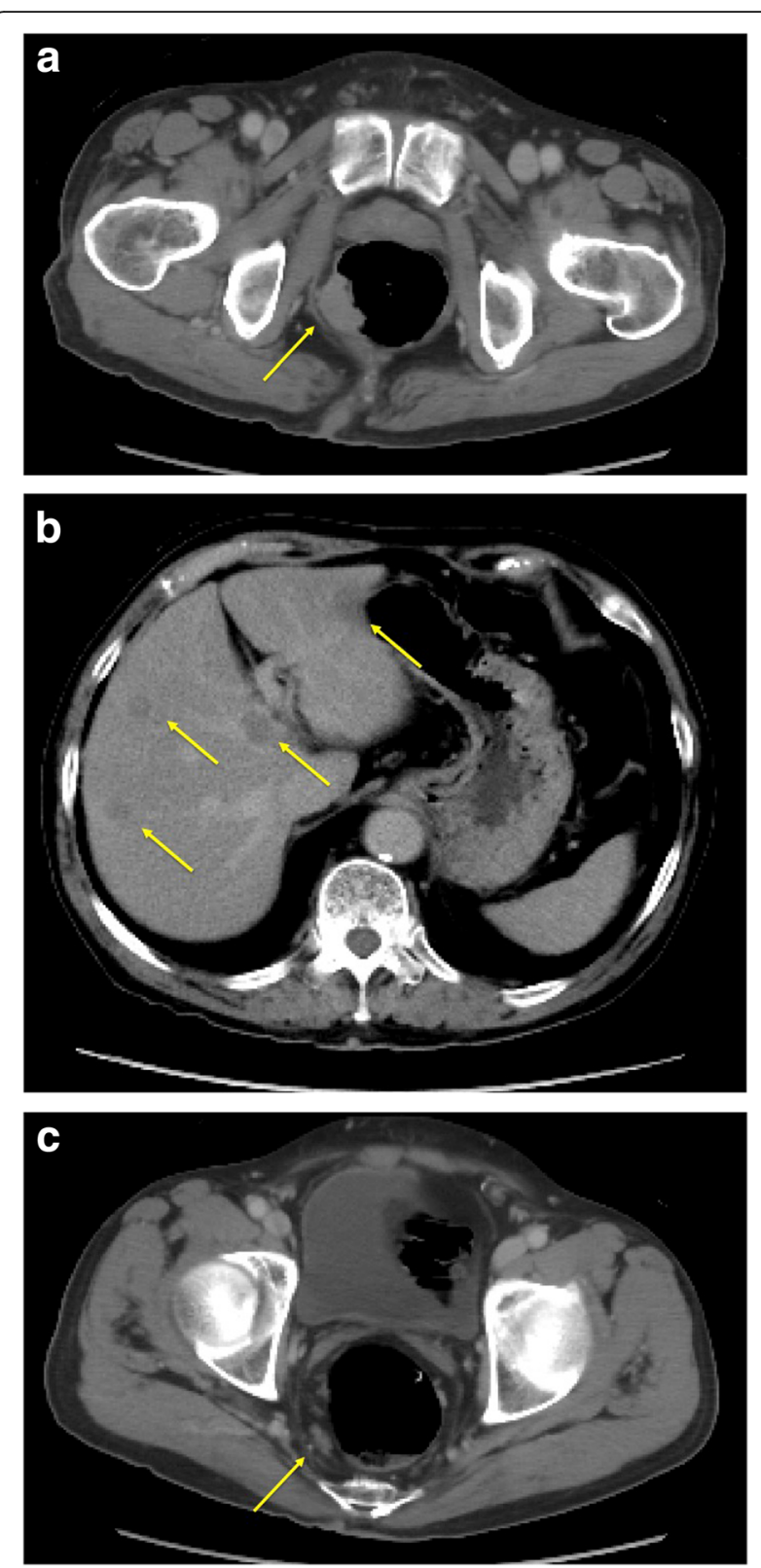

Fig. 2 Computed tomography (colonography) image. a This reveals a tumor in the lower rectum with suspected invasion of the right levator ani muscle. $\mathbf{b}, \mathbf{c}$ The paraintestinal lymph nodes are swollen, and there are multiple liver metastases

endocrine organs (Fig. 5). The patient was discharged on the tenth postoperative day.

Two months after surgery, the patient began chemotherapy with irinotecan $\left(60 \mathrm{mg} / \mathrm{m}^{2}\right)$ plus cisplatin $(60$ $\mathrm{mg} / \mathrm{m}^{2}$ ). In the second and third courses, each dose was reduced by $50 \%$ from the standard because of a renal dysfunction. After 3 courses, his liver metastases had increased in size, so we changed the regimen to carboplatin $\left(400 \mathrm{mg} / \mathrm{m}^{2}\right)$ plus etoposide $\left(100 \mathrm{mg} / \mathrm{m}^{2}\right)$. In the second course, each dose of carboplatin was reduced by 

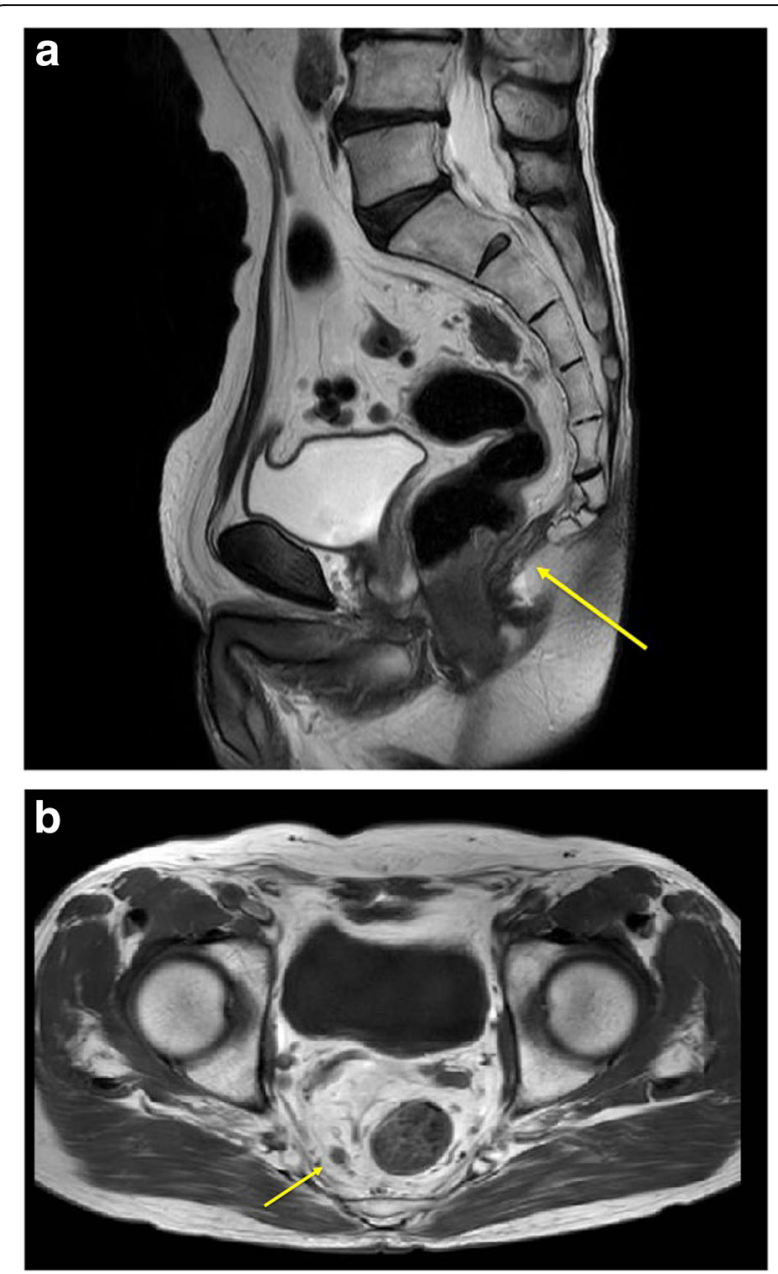

Fig. 3 Magnetic resonance imaging. a This reveals suspected tumor invasion of the right levator ani muscle. $\mathbf{b}$ The right lateral lymph nodes were swollen

$50 \%$ from the standard because of a febrile neutropenia. After 2 courses, the liver lesions continued to enlarge. At that point, we discontinued chemotherapy and provided supportive care. Twenty-seven months after surgery, he died of his disease.

Pathologic re-examination of the surgical specimen after he died revealed that the tumor was consistent with the 2019 WHO classification of NET G3.

\section{Discussion}

The incidence of neuroendocrine tumor was 1.09 per 100,000 persons in 1973 , increasing to 6.98 per 100 , 000 persons by 2012 in the USA [1]. The 2019 revised WHO classification of tumors of endocrine organs classifies grade 3 GEP-NENs into NET G3 and NEC G3 categories, indicating well- or poorly differentiated neoplasms, respectively. Cytologically, neuroendocrine tumors usually have abundant granular cytoplasm, which results in a low nuclear to
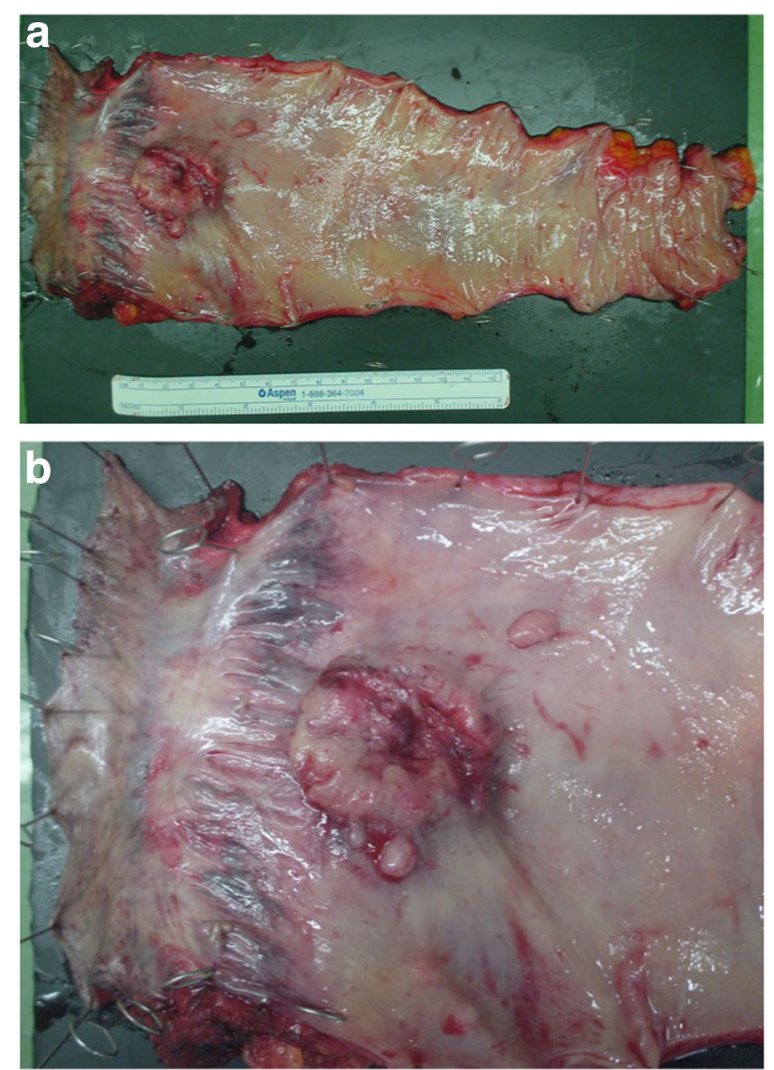

Fig. 4 Specimen: $\mathbf{a}, \mathbf{b}$ The tumor in the wall of the rectum measures $3.5 \times 2.8 \mathrm{~cm}$

cytoplasmic $(\mathrm{N} / \mathrm{C})$ ratio, and stippled chromatin. Cells of NEC G3 neoplasms have a lesser amount of granular cytoplasm and a higher $\mathrm{N} / \mathrm{C}$ ratio $[2,3]$. Immunohistochemically, both NET G3 and NEC G3 have a Ki-67 index of greater than $20 \%$ and an amitotic index of greater than 20 per 10 high-power fields. There are some differences in their pathologic characteristics. The rate of positive chromogranin A is $100 \%$ in NET G3 and $88.6 \%$ in NEC G3, with positive synaptophysin rates of $95.2 \%$ and $93.8 \%$, median Ki67-LI values of $28.5 \%$ and $80.0 \%$, loss of retinoblastoma protein $(\mathrm{Rb})$ expression in $0 \%$ and $54.5 \%$, presence of a KRAS gene mutation in $0 \%$ and $48.7 \%$, loss of $\mathrm{Rb}$ expression with a KRAS mutation in $0 \%$ and $30 \%$, and p53 expression in $0 \%$ and $75 \%$, respectively $[4,5]$. The expression of $\mathrm{Rb}$ and $\mathrm{p} 53$ is particularly useful especially in patients who are difficult to differentiate between NET G3 and NEC G3 because the expression rate is high in NEC G3, but neither are expressed in NET G3.

The primary treatment for GEP-NEN G3 is surgery. Tumors greater than $2 \mathrm{~cm}$ and those that invade the muscularis propria or with locoregional lymph node involvement should generally be managed similarly to 

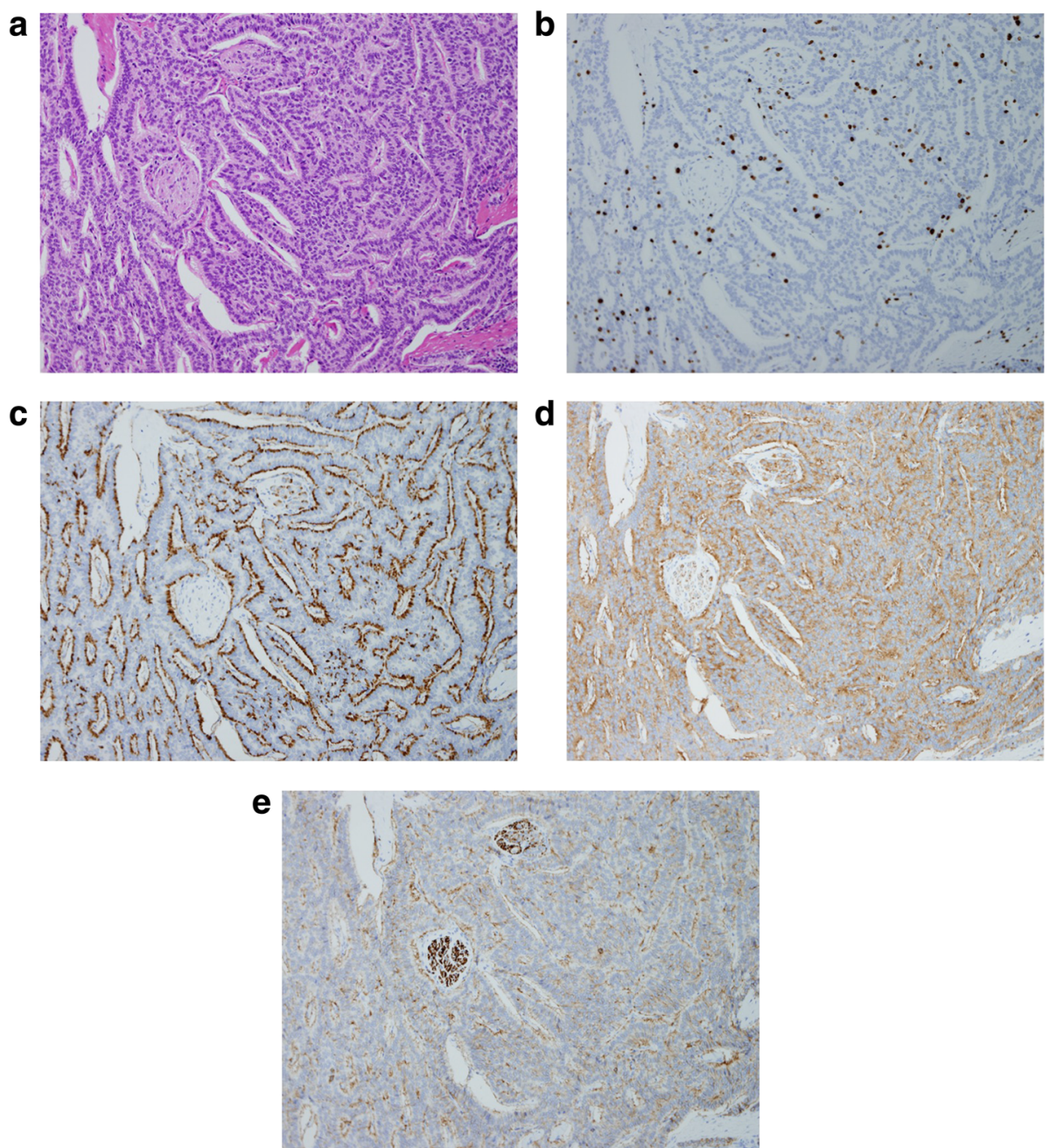

Fig. 5 Histopathologic findings. a Chromatin is increased in the atypical cells, which feature mitotic figures. Invasion involves the subserosa. Hematoxylin-eosin staining (magnification $\times 20$ ). b MIB-1 index $>20 \%$. c Positive staining for chromogranin A. $\mathbf{d}$ Positive staining for synaptophysin. e Positive staining for CD56

colorectal adenocarcinoma [6]. The high-grade malignancies NET G3 and NEC G3 have already developed distant metastases by the time of the primary resection, and even if the primary lesions are completely removed, they can recur on a long-term basis. For metastatic neuroendocrine carcinoma, the combination of cisplatin and etoposide is recommended as first-line therapy [6]. However, Hijioka et al. reported that NET G3 does not respond to platinum-based chemotherapy [4]. The Ki67 index is significantly higher in neuroendocrine carcinoma; Sorbye et al. and Heetfield et al. reported that patients with $\mathrm{Ki}-67$ less than $55 \%$ are less responsive to platinum-based chemotherapy $[7,8]$. There are currently no data on antitumor therapy for patients with metastatic colorectal NET G3. The Clinical Practice Guidelines in Oncology: Neuroendocrine Tumors Version 1, 2019, recommends octreotide, lanreotide, everolimus, and peptide receptor radionuclide therapy using $177 \mathrm{Lu}-$ dotatate, similar to that used for patients with grade 1 and grade 2 neuroendocrine tumors [9]. There is no known role for systemic adjuvant therapy. In this patient, we diagnosed neuroendocrine carcinoma based on the 2010 WHO classification, and he was treated with platinum-based chemotherapy. We could not change the diagnosis at that time, but we should not select platinum-based chemotherapy because the Ki-67 index of his tumor was $25 \%$ that means his tumor was less responsive to this therapy. Although his disease did not respond, he survived 17 months after he discontinued chemotherapy. His treatment course indicates that the tumor growth of NET G3 is slower than that of neuroendocrine carcinoma. Heetfield et al. reported that the median overall survival is 98.7 months for NET G3 and 17 months for neuroendocrine carcinoma [8].

In this case, we treated him as NEC till he died, and the diagnosis was changed due to the revision of the 
WHO classification in 2019. If he was diagnosed with NET G3 in WHO classification of 2019, he would be treated with the abovementioned therapy that the Clinical Practice Guidelines in Oncology recommended, and his prognosis might be extended [9].

We believe that it is important to distinguish between these 2 classifications to avoid using ineffective treatment modalities, and we should do pathologic reexamination when the disease does not respond for first chemotherapy or discontinued chemotherapy to give the appropriate treatment to NEC patients diagnosed by the WHO 2010 classification.

\section{Conclusions}

A definite diagnosis of NET G3 or NEC G3 is necessary to determine treatment strategy. Study of further case reports and case series should result in discovery of the optimal therapy.

\section{Abbreviations}

GEP-NEN G3: grade 3 gastroenteropancreatic neuroendocrine neoplasms; NET G3: Well-differentiated tumors; NEC G3: Poorly differentiated carcinomas; WHO: World Health Organization; CEA: Carcinoembryonic antigen; CA 19-

9: Cancer antigen 19-9; N/C: Nuclear-to cytoplasmic; EUS-guided

FNA: Endoscopic-guided fine-needle aspiration

\section{Acknowledgements}

None

\section{Authors' contributions}

$\mathrm{Ml}$ and $\mathrm{YH}$ wrote the manuscript. SY supervised the case. All other authors reviewed the manuscript. All authors read and approved the final

manuscript.

\section{Funding}

No funding was received for this case report

\section{Availability of data and materials}

Data sharing not applicable to this article since datasets were neither generated nor analyzed for the case series.

\section{Ethics approval and consent to participate}

Not applicable

\section{Consent for publication}

Written informed consent was obtained from the patient for the publication of this report.

\section{Competing interests}

The authors declare that they have no competing interests

Received: 3 April 2020 Accepted: 3 June 2020

Published online: 12 June 2020

\section{References}

1. Dasari A, Shen C, Halperin D, Zhao B, Zhou S, Xu Y, et al. Trends in the incidence, prevalence, and survival outcomes in patients with neuroendocrine tumors in the United States. JAMA Oncol. 2017:3:1335-42.

2. Tang LH, Basturk O, Jillian J, Klimstra DS. A practical approach to the classification of WHO grade 3 (G3) well differentiated neuroendocrine tumor (WD-NET) and poorly differentiated neuroendocrine carcinoma (PDNEC) of the pancreas. Am J Surg Pathol. 2016;40:1192-202.

3. Capano F, Grami O, Pugliese L, Pietrabissa A, Solsia E, Vanoli A. Grade 3 neuroendocrine tumor (G3 NET) in a background of multiple serotonin cell neoplasms of the ileum associated with carcinoid syndrome and aggressive behavior. Endocrine Pathol. 2018;29:369-73.
4. Hijioka S, Hosoda W, Matsuo K, Ueno M, Furukawa M, Yoshitomi H, et al. Rb loss and KRAS mutation are predictors of the response to platinum-based chemotherapy in pancreatic neuroendocrine neoplasm with grade 3: a Japanese multicenter pancreatic MEN-G3 study. Clin Cancer Res. 2017;23: 4635-2.

5. Konukiewitz B, Schlitter AM, Jesinghaus M, Pfister D, Steiger K, Segler A, et al. Somatostatin receptor expression related to TP53 and RB1 alterations in pancreatic and extrapancreatic neuroendocrine neoplasms with a Ki67index above 20\%. Mod Pathol. 2017:30:587-98.

6. Strosberg JR, Coppola D, Klimstra DS, Phan AT, Kulke MH, Wiseman GA, et al. The NANETS consensus guidelines for the diagnosis and management of poorly differentiated (high-grade) extrapulmonary neuroendocrine carcinomas. Pancreas. 2010;39:799-800.

7. Sorbye H, Welin S, Langer SW, Vestermark LW, Holt N, Osterlund P, et al. Predictive and prognostic factors for treatment and survival in 305 patients with advanced gastrointestinal neuroendocrine carcinoma (WHO G3): the NORDIC NEC study. Ann Oncol. 2013;24:152-60.

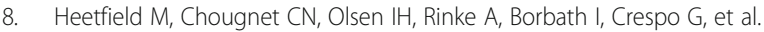
Characteristics and treatment of patients with G3 gastroenteropancreatic neuroendocrine neoplasms. Endocr Relat Cancer. 2015:22:657-64.

9. Clark OH, Benson AB, Berlin JD, Choti MA, Doherty GM, Engstrom PF, et al. NCC network: clinical practice guidelines in oncology: Neuroendocrine Tumors. Version 1.2019. https://www.nccn.org/professionals/physician_gls/ default.aspx. Accessed 2 June 2020.

\section{Publisher's Note}

Springer Nature remains neutral with regard to jurisdictional claims in published maps and institutional affiliations.

\section{Submit your manuscript to a SpringerOpen ${ }^{\circ}$ journal and benefit from:}

- Convenient online submission

- Rigorous peer review

- Open access: articles freely available online

- High visibility within the field

- Retaining the copyright to your article

Submit your next manuscript at $\boldsymbol{\nabla}$ springeropen.com 